\title{
New sweet cherry cultivars in intensive plantings
}

\author{
Szabó, Z. ${ }^{1}$, Farkas, E. ${ }^{2}$, Soltész, M. ${ }^{3}$, Fieszl, Cs. ${ }^{1}$, Balázs, G. ${ }^{1}$, Nyéki, J. ${ }^{1}$ \\ ${ }^{1}$ University of Debrecen Centre for Agricultural and Applied Economic Sciences, \\ H-4032 Debrecen, Böszörményi út 138., Hungary, E-mail: zszabo@agr.unideb.hu \\ ${ }^{2}$ Alma 2000 Ltd., 8911 Nagykutas, Almasétány 1., Hungary \\ ${ }^{3}$ Collage of Kecskemét, Faculty of Horticulture, H-6000 Kecskemét, Erdei Ferenc tér 1-3.
}

\begin{abstract}
Summary: The study took place in the largest sweet cherry plantation in West Hungary. The purpose has been the identification of those varieties, which will be suitable for intense cultivation, early fruiting and excellent fruit quality, moreover, the selection of the optimal phytotechnical procedures. At the same time, scion-rootstock combinations have been tested also from the point of view of growing intensity and fruiting in high-density plantation. The dense planting is induced to start fruiting early and yield regularly by special methods. Yielding was stimulated by maintaining the balance of vegetative-generative growth by binding the shoots, by summer pruning, by cuts on the trunk and root pruning. Best experiences have been found in yield and quality in the following varieties: Canada Giant, Carmen, Firm Red, Giant Red, Katalin, Kordia, Regina. Dense planting has been feasible also on vigorous rootstock, like P. mahaleb. Dwarfing rootstocks like P-HL-A, Gisela 6, accelerate the formation of flower buds and yielding earlier with fruits of adequate size. 'Firm Red' and 'Giant Red' excelled with their large fruit (>27 mm diameter) in all combinations, thus being promising under Hungarian conditions.
\end{abstract}

Keywords: cherry, variety, rootstock, training system, pruning

\section{Introduction}

In developed countries, the intensity of fruit growing practices grew significantly during the last 2-3 decennia. In countries, where land and man power are expensive, the high density plantations are preferred.

In Italy, growing systems applied are highly variable as presented by Lugli \& Musacchi (2009). Climatic conditions and marketing require in other countries special growing constructions for fruit plantations. Conditions and possibilities are summarised for a high tunnel cherry production system by Lang (2009).

In Hungary, Soltész et al. (2000) surveyed the possible growing systems applied in fruit growing. A comprehensive study of the possible cherry growing systems is available since Hrotkó et al. (2008), who dealt also with the training of young trees. Gonda et al. (2007) developed the crown forms and the respective pruning systems for the new Hungarian cherry varieties recommended in high density plantations.

The volume of cherry grown in Hungary declined during the last two decennia by $40-50 \%$. At the moment, plantations of 2000 ha produce yearly 10000 tons of cherries. Home consumption and export possibilities justify the establishment of new cherry plantings. The economics as well as adequate fruit quality of cherry production is bound exclusively to the intense high density planting systems. The purpose of the present study is to find the right sweet cherry varieties, which are productive and promise excellent quality, and at the same time we endeavour to develop the variety specific growing technologies.

\section{Materials and methods}

The plantation examined is in the Western Hungarian commune, Nagykutas, $230 \mathrm{~m}$ above sea level. Meteorological data are: yearly mean temperature: $101^{\circ} \mathrm{C}$, precipitation $693 \mathrm{~mm}$, number of sunny hours 1927.

The brown forest soil has a favourable water husbandry, and keeps well soil humidity being sandy loam with low acidity. Pruning was performed twice, in the winter and after harvest.

The date of the commercial plantations were 1999 and 2003, on P.mahaleb seedling, the planting design was $3.5 \times 1.0$ and $4 \times 1.1 \mathrm{~m}$, the trees were trained to slender spindle. The commercial yield of the plantation amounted $2-3 \mathrm{t} /$ ha in the $3^{\text {rd }}$ year already and attained the maximum with the $7^{\text {th }}$ year. Occasionally, 20 t/ha yields occurred. The experiment for testing rootstocks was planted is 2006, using P. mahaleb, P-HL-A, Gisela 6, Ma×Ma 14, planted to $3.5 \times 1.0 \mathrm{~m}$ distance with Giant Red, Firm Red varieties. Properties as tree size, blooming date, flower density, fruit load, yield, fruit quality have been registered.

\section{Results and discussion}

\section{Evaluation of the varieties}

In Hungary, the leading sweet cherry varieties are: Germersdorfi óriás, Bigarreau Burlat, Katalin, Van and Linda. In recent plantations appear some new Hungarian 
(Carmen and Vera) and foreign (Sweet Heart, Firm Red and Giant Red) varieties.

Varieties supposed to become important in Hungary are tested under conditions of intense technologies. Their phenological phases of bloom and harvest periods, yields and fruit characteristics are registered.

Regarding the date of blooming periods, the differences between varieties did not surpass a 3-4 days' period, the times of blooming largely overlapped each other in the assortment. As a rare exception, in 2008, weather conditions prolonged the beginning of bloom up to 13 days. The blooming period lasted 5-16 days. During the next three years, the start of bloom between the earliest and latest blooming varieties occurred within 5 days. The blooming period lasted more than 10 days in the earliest starting varieties as a consequence of the cool spring of 2008. In the following seasons, the blooming periods of varieties were medium long and differed less from each other. In 2010, the cool and wet weather lengthened the blooming period to 14-20 days (Table 1).

Dates of ripening varied yearly between the seasons along a period of 10-15 days, and also the sequence of varieties might change. In Figure 1, overage data of ripening are shown. From the point of view of the market, varieties producing fruits with a diameter more than $28 \mathrm{~mm}$ are considered to be of interest. Among the traditional varieties, Canada Giant, Katalin and Regina are suitable, among the new ones Firm Red and Giant Red (Table 2). Except Canada Giant, the content of soluble solids in the fruit was high enough.

\section{Evaluation of the rootstocks}

In Hungary, Prunus mahaleb as a rootstock for cherry trees is generally utilised. Grafts are made on P. mahaleb at a rate of $80-90 \%$ as well as in the plantation examined. The way to moderate growing vigour is relied essentially to root pruning.

Growing intensity of Firm Red and Giant Red varieties was similar on the four different rootstocks examined. Nevertheless, most vigour was observed on grafts using $P$. mahaleb and on MaxMa 14, whereas the lowest size was experienced on Gisela 6 rootstocks (Table 3). Those trees needed a supporting system, absolutely.

Rootstocks are decisive in determining the date of bloom as well as the intensity of blooming (Table 4). Higher vigour is combined with later bloom and lower flower density especially in the first fruiting year.

The early and intense flower production of trees grafted to Gisela 6 was outstanding. As a result, fruiting began earlier on those trees, consequently fruit thinning is soon actual. In 2010 , flower density was mediocre, all the same, fruit set was poor on the trees grafted on P. mahaleb.

The mean diameter of fruits was larger than $27 \mathrm{~mm}$ in all combinations. Largest fruits were harvested in the first year of the experiment (2008). In Giant Red, more than $30 \mathrm{~mm}$, in Firm Red, more than $28 \mathrm{~mm}$ diameters are measured (Table 5). In the content of soluble solids, no significant differences are produced by the rootstocks, on the contrary, seasonal effects were distinct.

Table 1: Blooming dates of sweet cherry varieties at Nagykutas (2008-2011)

\begin{tabular}{|c|c|c|c|c|c|c|c|c|}
\hline \multirow[b]{2}{*}{ Variety } & \multicolumn{2}{|c|}{2008} & \multicolumn{2}{|c|}{2009} & \multicolumn{2}{|c|}{2010} & \multicolumn{2}{|c|}{2011} \\
\hline & Start of bloom & $\begin{array}{c}\text { Length of } \\
\text { blooming period } \\
\text { (days) }\end{array}$ & Start of bloom & $\begin{array}{c}\text { Length of } \\
\text { blooming period } \\
\text { (days)) }\end{array}$ & Start of bloom & $\begin{array}{c}\text { Length of } \\
\text { blooming period } \\
\text { (days)) }\end{array}$ & Start of bloom & $\begin{array}{c}\text { Length of } \\
\text { blooming period } \\
\text { (days)) }\end{array}$ \\
\hline Aida & April 06 & 8 & April 08. & 14 & April 07. & 16 & April 07. & 7 \\
\hline Alex & April 05. & 10 & April 08. & 13 & April 07. & 18 & April 09. & 8 \\
\hline Canada Giant & April 03. & 10 & April 10. & 12 & April 08. & 18 & April 10. & 10 \\
\hline Carmen & April 09. & 10 & April 09. & 14 & April 07. & 17 & April 09. & 10 \\
\hline Celeste & March 31 & 10 & April 08. & 9 & - & - & - & - \\
\hline Chelan & March 29 & 16 & April 09. & 11 & - & - & - & - \\
\hline Cristalina & April 04 & 9 & April 09. & 12 & April 08. & 15 & April 05. & 9 \\
\hline Ferrovia & April 05. & 9 & April 09. & 8 & - & - & - & - \\
\hline Firm Red $*$ & April 05. & 15 & April 08. & 14 & April 09. & 15 & April 06, & 10 \\
\hline Germesdorfi 3 & April 03. & 16 & April 09. & 12 & April 09. & 18 & April 09. & 9 \\
\hline Giant Red * & April 05. & 12 & April 09. & 13 & April 08. & 15 & April 08, & 9 \\
\hline Katalin & April 01. & 16 & April 08. & 14 & - & - & - & - \\
\hline Kordia & April 03. & 10 & April 08. & 11 & April 09. & 14 & April 07. & 12 \\
\hline Krupnoplodnaja & April 11. & 8 & April 09. & 13 & April 09. & 20 & April 10. & 8 \\
\hline Lambert & April 08. & 8 & April 08. & 13 & April 08. & 17 & April 07. & 9 \\
\hline Linda & April 09. & 10 & April 08. & 12 & April 08. & 15 & - & - \\
\hline
\end{tabular}

*Remark: Firm Red and Giant Red are grafted ont Maxma 14 rootstocks, whereas the rest is grown on P. mahaleb seedling roots 
Table 2: Diameter, weigth and soluble solids content of cherry fruits (Nagykutas, 2008-2011)

\begin{tabular}{|c|c|c|c|c|c|c|c|c|c|c|c|}
\hline \multirow[b]{2}{*}{ Variety } & \multicolumn{2}{|c|}{2008} & \multicolumn{3}{|c|}{2009} & \multicolumn{3}{|c|}{2010} & \multicolumn{3}{|c|}{2011} \\
\hline & $\begin{array}{l}\text { Diameter } \\
(\mathrm{mm})\end{array}$ & $\begin{array}{l}\text { Mass of } \\
\text { fruits }(\mathrm{g})\end{array}$ & $\begin{array}{l}\text { Diameter } \\
\text { of fruits } \\
(\mathrm{mm})\end{array}$ & $\begin{array}{l}\text { Mass } \\
\text { of fruits } \\
(\mathrm{g})\end{array}$ & $\begin{array}{c}\text { Soluble } \\
\text { solids } \\
\text { content }(\%)\end{array}$ & $\begin{array}{c}\text { Diameter } \\
\text { of fruits } \\
(\mathrm{mm})\end{array}$ & $\begin{array}{c}\text { Mass } \\
\text { of fruits } \\
(\mathrm{g})\end{array}$ & $\begin{array}{c}\text { Soluble } \\
\text { solids } \\
\text { content }(\%)\end{array}$ & $\begin{array}{c}\text { Diameter } \\
\text { of fruits } \\
(\mathrm{mm})\end{array}$ & $\begin{array}{l}\text { Mass } \\
\text { of fruits } \\
(\mathrm{g})\end{array}$ & $\begin{array}{c}\text { Soluble } \\
\text { solids } \\
\text { content }(\%)\end{array}$ \\
\hline Aida & 21,5 & 5,9 & 24,1 & 6,8 & 16,3 & 24,5 & 7,8 & 13,9 & 25,2 & 7,9 & 17,2 \\
\hline Alex & - & - & 24,9 & 8,1 & 19,6 & 23 & 6,9 & 14,9 & - & - & - \\
\hline Canada Giant & 27,4 & 9,4 & 26,2 & 9,5 & 13,8 & 26,2 & 8,2 & 13,3 & 27,7 & 11,1 & 14,0 \\
\hline Carmen & 30,9 & 14,6 & 25,2 & 8,5 & 12,7 & 26,1 & 9,5 & 12,7 & - & - & - \\
\hline Celeste & 28,3 & 9,9 & 25,2 & 6,8 & 12,2 & - & - & - & - & - & - \\
\hline Chelan & 23,1 & 6,8 & 23,7 & 7,5 & 13,7 & - & - & - & - & - & - \\
\hline Cristalina & 27 & 9,3 & - & - & - & 25,3 & 7,8 & 17,9 & 26,1 & 9,8 & 13,8 \\
\hline Ferrovia & 28 & 10,4 & 23,9 & 7,8 & 14,2 & - & - & - & - & - & - \\
\hline Firm Red $*$ & 28,1 & 9,5 & 28,2 & 9,4 & 17,3 & 28,2 & 9,1 & 17 & 30,7 & 11,7 & 16,8 \\
\hline Germesdorfi 3 & - & - & 25 & 8,7 & 15,5 & 26,3 & 8,7 & 13,5 & 26,6 & 9,2 & 12,7 \\
\hline Giant Red * & 30,3 & 12,8 & 28,1 & 9,4 & 18,9 & 27,1 & 10 & 17,1 & 31,4 & 13,7 & 17,1 \\
\hline Katalin & 26,2 & 10,4 & 27,1 & 9,9 & 19,8 & 27 & 10,2 & 18,6 & - & - & - \\
\hline Kordia & 24,6 & 8,3 & - & - & - & 26,9 & 10 & 16,7 & - & - & - \\
\hline Krupnoplodnaja & 24,3 & 7 & 23,6 & 7,5 & 17,9 & 23,7 & 7,3 & 15,1 & 27,0 & 9,8 & 16,9 \\
\hline Lambert & 27,2 & 9,7 & 30,4 & 11,9 & 14,6 & 25,4 & 8,8 & 16,6 & 28,8 & 11,4 & 15,9 \\
\hline Linda & 23,7 & 7,2 & 23,5 & 6,8 & 16,3 & 25,7 & 9,2 & 17,9 & 27,4 & 10,4 & 15,1 \\
\hline Regina & 26,7 & 10,5 & 28,6 & 12,5 & 16,4 & 27,8 & 11,3 & 17,8 & 31,3 & 15,0 & 18,5 \\
\hline Sandra Rose & 30,4 & 11,4 & 26,3 & 9,4 & 18,2 & - & - & - & - & - & - \\
\hline Santina & 22,8 & 6,5 & 25,1 & 9 & 16,3 & 25,2 & 7,9 & 14,9 & 24,9 & 7,6 & 16,9 \\
\hline Skeena & 26,2 & 9,1 & 24,7 & 8,7 & - & 26,3 & 9,7 & 16,3 & - & - & - \\
\hline Sunburst & 26,9 & 9,2 & 27,2 & 10,8 & 16,2 & - & - & - & - & - & - \\
\hline Sweet Early & - & - & 26,9 & 9,7 & - & 24,4 & 7,8 & 11,5 & - & - & - \\
\hline Sweet Heart & - & - & - & - & 16,7 & 23,4 & 7,8 & 15,3 & - & - & - \\
\hline Sympfony & 23,1 & 6,9 & 23,9 & 7,8 & 14,2 & - & - & - & - & - & - \\
\hline Techlovan & 29,5 & 14,4 & 25,7 & 9 & 11,9 & - & - & - & - & - & - \\
\hline Vera & 26,2 & 8,8 & 25 & 8 & 13,7 & 27,4 & 9,7 & 12,5 & 27,7 & 10,4 & 19,2 \\
\hline
\end{tabular}

*Remark: Firm Red and Giant Red are grafted ont Maxma 14 rootstocks, whereas the rest is grown on P. mahaleb seedling roots

Table 3: Three size of sweet cherry trees grown on different rootstocks (Nagykutas, 2010)

\begin{tabular}{|c|c|c|c|c|c|c|c|c|}
\hline \multirow{3}{*}{ Rootstock } & \multicolumn{4}{|c|}{ Giant Red } & \multicolumn{4}{|c|}{ Firm Red } \\
\hline & \multirow{2}{*}{$\begin{array}{l}\text { Girth of the } \\
\text { trunk }(\mathrm{cm})\end{array}$} & \multirow{2}{*}{$\begin{array}{l}\text { Height of the } \\
\text { tree }(\mathrm{m})\end{array}$} & \multicolumn{2}{|c|}{ Diameter of the crown $(\mathrm{m})$} & \multirow{2}{*}{$\begin{array}{l}\text { Girth of the } \\
\text { trunk }(\mathrm{cm})\end{array}$} & \multirow{2}{*}{$\begin{array}{l}\text { Height of the } \\
\text { tree }(m)\end{array}$} & \multicolumn{2}{|c|}{ Diameter of the crown (m) } \\
\hline & & & $\begin{array}{l}\text { At the row } \\
\text { length }\end{array}$ & $\begin{array}{l}\text { Perpendicularly } \\
\text { to the row }\end{array}$ & & & $\begin{array}{c}\text { At the row } \\
\text { length }\end{array}$ & $\begin{array}{l}\text { Perpendicularly } \\
\text { to the row }\end{array}$ \\
\hline P. mahaleb & 22.4 & 2.7 & 2.0 & 1.7 & 25.3 & 3.1 & 2.0 & 2.0 \\
\hline Maxma 14 & 24.6 & 3.0 & 1.6 & 1.7 & 24.5 & 3.1 & 2.0 & 1.6 \\
\hline P-HL-A & 23.6 & 3.1 & 1.8 & 1.5 & 22.4 & 3.1 & 1.9 & 1.6 \\
\hline Gisela 6 & 21.0 & 2.9 & 1.5 & 1.7 & 18.7 & 2.8 & 1.6 & 1.5 \\
\hline
\end{tabular}


Table 4: Beginning of bloom and flower density of sweet cherry varieties (Nagykutas, 2008-2011)

\begin{tabular}{|c|c|c|c|c|c|c|c|c|c|c|c|c|c|c|c|c|}
\hline \multirow{3}{*}{ Rootstock } & \multicolumn{8}{|c|}{ Giant Red } & \multicolumn{8}{|c|}{ Firm Red } \\
\hline & \multicolumn{2}{|c|}{2008} & \multicolumn{2}{|c|}{2009} & \multicolumn{2}{|c|}{2010} & \multicolumn{2}{|c|}{2011} & \multicolumn{2}{|c|}{2008} & \multicolumn{2}{|c|}{2009} & \multicolumn{2}{|c|}{2010} & \multicolumn{2}{|c|}{2011} \\
\hline & $\begin{array}{c}\text { Start of } \\
\text { bloom }\end{array}$ & $\begin{array}{c}\text { Flower } \\
\text { density } \\
(0-5)\end{array}$ & $\begin{array}{c}\text { Start of } \\
\text { bloom }\end{array}$ & $\begin{array}{c}\text { Flower } \\
\text { density } \\
(0-5)\end{array}$ & $\begin{array}{c}\text { Start of } \\
\text { bloom }\end{array}$ & $\begin{array}{c}\text { Flower } \\
\text { density } \\
(0-5)\end{array}$ & $\begin{array}{c}\text { Start of } \\
\text { bloom }\end{array}$ & $\begin{array}{c}\text { Flower } \\
\text { density } \\
(0-5)\end{array}$ & $\begin{array}{l}\text { Start of } \\
\text { bloom }\end{array}$ & $\begin{array}{c}\text { Flower } \\
\text { density } \\
(0-5)\end{array}$ & $\begin{array}{c}\text { Start of } \\
\text { bloom }\end{array}$ & $\begin{array}{c}\text { Flower } \\
\text { density } \\
(0-5)\end{array}$ & $\begin{array}{c}\text { Start of } \\
\text { bloom }\end{array}$ & $\begin{array}{c}\text { Flower } \\
\text { density } \\
(0-5)\end{array}$ & $\begin{array}{c}\text { Start of } \\
\text { bloom }\end{array}$ & $\begin{array}{c}\text { Flower } \\
\text { density } \\
(0-5)\end{array}$ \\
\hline P. mahaleb & April 5. & 2 & $\begin{array}{c}\text { April } \\
09 .\end{array}$ & 2,4 & $\begin{array}{c}\text { April } \\
08 .\end{array}$ & 3,4 & $\begin{array}{c}\text { April } \\
07 .\end{array}$ & 2,8 & April 5. & 2 & $\begin{array}{c}\text { April } \\
08 .\end{array}$ & 2,7 & $\begin{array}{c}\text { April } \\
09 .\end{array}$ & 4,1 & $\begin{array}{c}\text { April } \\
07 .\end{array}$ & 3,2 \\
\hline Maxma 14 & April 8. & 2 & $\begin{array}{c}\text { April } \\
08 .\end{array}$ & 2 & $\begin{array}{c}\text { April } \\
07 .\end{array}$ & 3,7 & $\begin{array}{c}\text { April } \\
08 .\end{array}$ & 2,6 & April 6. & 2 & $\begin{array}{c}\text { April } \\
08 .\end{array}$ & 2,2 & $\begin{array}{c}\text { April } \\
07 .\end{array}$ & 4,3 & $\begin{array}{c}\text { April } \\
06 .\end{array}$ & 3,7 \\
\hline P-HL-A & April 4. & 3 & $\begin{array}{c}\text { April } \\
08 .\end{array}$ & 2,9 & $\begin{array}{c}\text { April } \\
08 .\end{array}$ & 4,5 & $\begin{array}{c}\text { April } \\
06 .\end{array}$ & 2,8 & April 5. & 3 & $\begin{array}{c}\text { April } \\
08 .\end{array}$ & 2,7 & $\begin{array}{c}\text { April } \\
08 .\end{array}$ & 3,9 & $\begin{array}{c}\text { April } \\
06 .\end{array}$ & 3,4 \\
\hline Gisela 6 & April 3. & 5 & $\begin{array}{c}\text { April } \\
08 .\end{array}$ & 4,3 & $\begin{array}{c}\text { April } \\
07 .\end{array}$ & 3,5 & $\begin{array}{c}\text { April } \\
07 .\end{array}$ & 4 & April 2. & 5 & $\begin{array}{c}\text { April } \\
09 .\end{array}$ & 4,3 & $\begin{array}{c}\text { April } \\
07 .\end{array}$ & 4,2 & $\begin{array}{c}\text { April } \\
07 .\end{array}$ & 4,1 \\
\hline
\end{tabular}

\begin{tabular}{|c|c|c|c|c|c|c|c|c|c|c|c|}
\hline \multirow{2}{*}{ Variety } & \multicolumn{3}{|c|}{ May } & \multicolumn{6}{|c|}{ June } & \multicolumn{2}{|c|}{ July } \\
\hline & 15 & 20 & 25 & 31 & 5 & 10 & 15 & 20 & 25 & 30 & 5 \\
\hline \multicolumn{12}{|l|}{ Sweet Early } \\
\hline \multirow{2}{*}{\multicolumn{12}{|c|}{$\begin{array}{l}\text { Carmen } \\
\text { Chelan }\end{array}$}} \\
\hline \multirow{2}{*}{\multicolumn{12}{|c|}{$\begin{array}{l}\text { Chelan } \\
\text { Linda }\end{array}$}} \\
\hline & & & & & & & & & & & \\
\hline \multicolumn{12}{|l|}{ Aida } \\
\hline \multicolumn{12}{|l|}{ Vere } \\
\hline \multirow{2}{*}{\multicolumn{12}{|c|}{$\begin{array}{l}\text { Firm Red } \\
\text { Celeste }\end{array}$}} \\
\hline & & & & & & - & $\bar{\square}$ & & & & \\
\hline \multicolumn{12}{|l|}{ Giant Red } \\
\hline \multicolumn{12}{|l|}{ Techlovan } \\
\hline \multirow{2}{*}{\multicolumn{12}{|c|}{$\begin{array}{l}\text { Santa Rose } \\
\text { Santina }\end{array}$}} \\
\hline & & & & & & & & & & & \\
\hline \multicolumn{12}{|l|}{ Germesrdorfi 3} \\
\hline \multicolumn{12}{|l|}{ Lambert } \\
\hline \multicolumn{12}{|l|}{ Krupnoplodnaj } \\
\hline \multirow{2}{*}{\multicolumn{12}{|c|}{ Sunburst }} \\
\hline & & & & & & & & & & & Canada Gia \\
\hline \multicolumn{12}{|l|}{ Cristalina } \\
\hline Ferrovie & & & & & & & & & $E$ & & \\
\hline \multicolumn{12}{|l|}{$\begin{array}{l}\text { Sympfony } \\
\text { Alex }\end{array}$} \\
\hline Alex & & & & & & & & & & - & \\
\hline \multicolumn{12}{|l|}{ Sweet Heart } \\
\hline Katalin & & & & & & & & & $=$ & & $=$ \\
\hline Skeena & & & & & & & & & & & \\
\hline Regina & & & & & & & & & & & \\
\hline
\end{tabular}

Figure 1: Maturity chart

\section{Conclusion}

Adaptation to novel marketing possibilities suggests an extension of super intensive growing practices. For that purpose, varieties with large fruit size and early fruiting are needed.

Dense planting has been feasible also on vigorous rootstock, like $P$. mahaleb. Dwarfing rootstocks like PHL-A, Gizela 6, accelerate the formation of flower buds and yielding earlier with fruits of adequate size. 'Firm Red' and 'Giant Red' excelled with their large fruit $(>27 \mathrm{~mm}$ diameter) in all combinations, thus being promising under Hungarian conditions.
Table 5: Fruit quality of sweet cherry trees grown on different rootstocks (Nagykutas, 2008-2011)

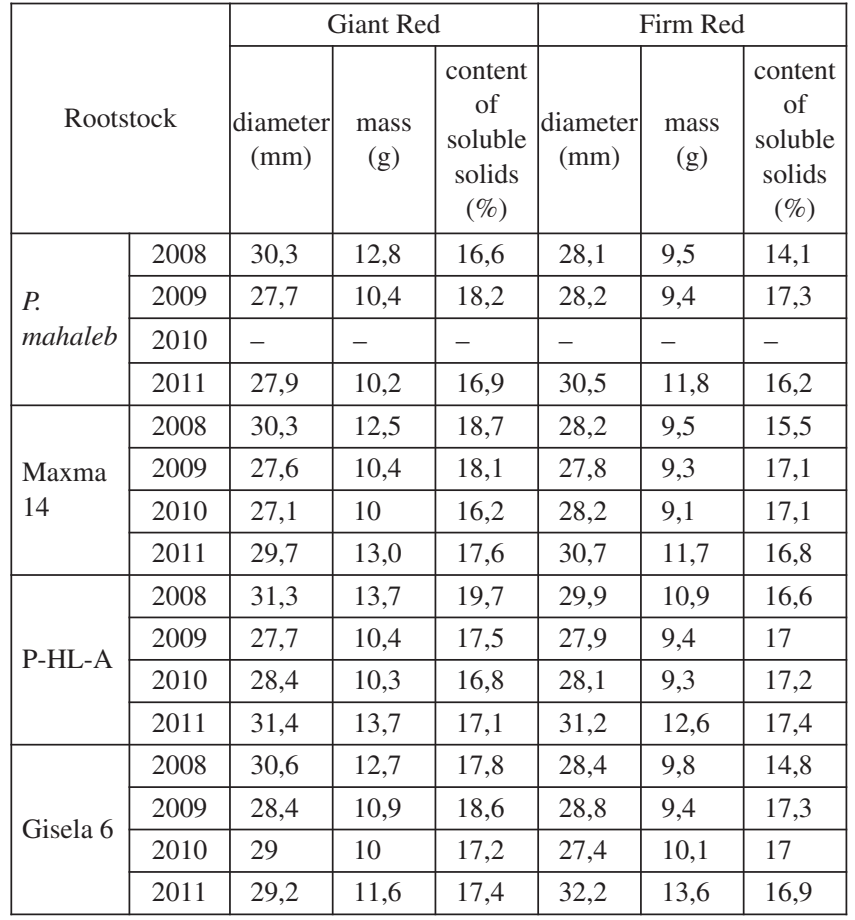

\section{Aknowledgement}

Research was sponsored by NFÜ (project number: TECH_08-A3/2-2008-0373)

\section{References}

Gonda, I., Király, K. \& Holb, I. (2007): Examination of growth of cherry cultivars adapted to intensive production. Acta Horticulturae, 732: 429-434.

Hrotko, K., Magyar, L., Gyeviki, M. \& Simon, G. (2008): Sistemi di allevamento e potatura per impianti ad alta densitá: le esperienze dell' Ungheria. Frutticoltura 70 (3): 10-18.

Lang, G. A. \& Meland, M. (2009): High tunnel production systems for the late season sweet cherry market. $6^{\text {th }}$ International Cherry symposium. November 15-19. Renoca-Vina del Mar, Chile. Abstract Book 18.

Lugli, S. \& Musacchi, S. (2009): L'evoluzione delle forme di allevamento nella cerasicolture specializzata. Convegno „Il ciliego ad alta densita': il futuro a portata di mano", Ferrara 5 Giugno

Soltész, M., Szabó, Z. \& Nyéki, J. (2000): Training systems of fruit trees in Hungary. International Journal of Horticultural Science 6, (1): 123-127. 